\title{
Fin Rudder Roll Stabilisation of Ships: a Gain Scheduling Control Methodology
}

\author{
Hervé Tanguy and Guy Lebret
}

\begin{abstract}
Active control of ship roll is necessary for operability of an important number of ships. As such it has been strongly developed in the past twenty years. Taking into account the variations of the environment is a means of improving performances. The ship behaviour is modeled as a MIMO LPV system; a methodology is presented which leads to a gain-scheduled control law. Synthesis is based on multi-objective optimisation and polytopic representation of the standard system, which depends on ship speed and on a stabilisation quality factor. Simulation results are given.
\end{abstract}

\section{INTRODUCTION}

Sea-keeping abilities determines the use of numerous ships such as military vessels (operability, aircraft landing, crew comfort) and passager vessels for passengers' comfort and security. Attenuating motions, especially roll, pitch and heave, by passive and much more by active stabilisation systems drastically improves the operability.

A major improvment in the stabilisation system performances should be to adapt to the environmental conditions: waves (encounter angle, power, dominant frequency), ship speed, loading conditions. However, there is relatively few published documents on such control laws. Yet, the dependance on the ships speed has been used for many years [1].

This document details the investigation realised for roll stabilisation towards the use of information about the environment. The context aims to be as realistic as possible, and is based on industrial data. A methodology is proposed, which leads to a gain scheduled controller based on $H_{\infty} / L M I$ results [2].

The paper is organized as follows: the process and its environment are described in section 2 as a natural MIMO linear parameterically varying (LPV) system. The control methodology is detailed in section 3. A four steps methodology leads to the gain scheduled controller. It is applied in section 4 on a frigate type vessel and the obtained performances are compared to those of invariant PID or $H_{\infty}$ controllers. Section 5 gives perspectives of improvement.

\section{MODEL}

This section aims at showing that a ship in a seaway can be modelled as a linear parameterically varying system.

H. Tanguy (sirehna@sirehna.ec-nantes.fr) was with Institut de Recherche en Communications et Cybernétique de Nantes (IRCCyN) and SIREHNA, now with SIREHNA, Nantes, France

G. Lebret (guy.lebreteirccyn.ec-nantes.fr) is with IRCCyN, Nantes, France
Comprehensive models derived from hydrodynamics lead to non linear partial derivative equations. Acceptable simplifying assumptions are made: amplitude of motions are small; the ship dynamics is independent of the swell frequency; the contribution of sea perturbation to motions in the sensors signal is linear.

This paragraph is a compact but also a slightly more detailed version of a paragraph of [3] (see also [4], [5]).

\section{A. Rigid-Body equation of motion}

In a Body-fixed frame, with origine corresponding to the average position of the center of mass, the equation of the motion is

$$
M(\eta) \ddot{\eta}+C(\eta, \dot{\eta}) \dot{\eta}=\tau
$$

where $\eta=\left(\begin{array}{lll}y & \phi & \psi\end{array}\right)^{\top}$ gives the position of the center of mass. The sway $(y)$, roll $(\phi)$ and yaw $(\psi)$ motions are supposed (this is realistic) to be decoupled from the others degree of freedom, surge $(x)$, heave $(z)$ and pitch $(\theta)$.

The external forces $(\tau)$ are reduced to the sum of hydrodynamics efforts $\left(\tau_{H}\right)$ due to the waves and of actuators efforts $\left(\tau_{A}\right)$. Their description ( $\S$ II-C and II-D) will lead to the final complete models of motion ( $\S$ II-E). The propulsion problem is not addressed in this work, and it is assumed that the ship speed is controlled by another manner.

\section{B. Sea disturbance}

Waves are the result of the sustained action of the wind over a wide sea surface. Complex sea states are considered to be the superposition of an infinite number of monochromatic waves, distributed in all directions.

Wave amplitude spectra, allow to characterise sea states all over the planet. The following spectrum, called Modified Pierson-Moskowitz [4], will be used in the simulations

$$
S_{W}(\omega)=\frac{4 \pi^{3} H_{s}^{2}}{T_{z}^{4} \omega^{5}} \exp \left(\frac{-16 \pi^{3}}{T_{z}^{4} \omega^{4}}\right)
$$

where $H_{s}$ and $T_{z}$ characterise the sea state, and correspond to the wave height and the mean zero-crossing period.

Due to Doppler effect, the wave frequency of a monochromatic wave observed from a moving ship is different from the one seen by a motionless observer. The encounter frequency is given by

$$
\omega_{e}=\omega\left(1-\omega \frac{V}{g} \cos \left(\psi_{e}\right)\right)
$$

where $\psi_{e}$ is the angle between the ship motion direction and the wave propagation direction. Then, a monochromatic wave seen from the moving ship has a pulsation which is 
a function of its true pulsation $\omega$, the encounter angle $\psi_{e}$ and the speed of the ship $V$.

\section{Hydrodynamics forces}

With the assumptions of small motions and of a monochromatic wave, it is possible to separate the hydrodynamics forces $\tau_{H}$ into three different types:

- $\tau_{B}$ : the buoyancy efforts (from Archimedes' principle),

- $\tau_{R}$ : the radiation forces; they are the applied forces on a moving ship on a calm sea,

- $\tau_{W}$ : the incident and diffracted waves forces; they are the applied forces due to the waves on a motionless ship.

$$
\tau_{H}=\tau_{B}+\tau_{R}+\tau_{W}
$$

and with the previous definitions (2) and (3), and assuming that the buoyancy forces depend linearly on the ship's attitude,

$$
\begin{aligned}
\tau_{B} & =-G \eta \\
\tau_{R} & =-M_{A}\left(\omega, \psi_{e}, V\right) \ddot{\eta}-D_{A}\left(\omega, \psi_{e}, V\right) \dot{\eta} \\
\tau_{W}(t) & =\int_{0}^{t} K_{W}(\tau) \zeta(t-\tau) d \tau
\end{aligned}
$$

where $K_{W}$ is the convolution kernel of the waves forces ${ }^{1}$ and $\zeta(t)-$ the height of the sea surface - is obtained from the spectrum (2).

One point to note here is that $\tau_{R}$ is a function of $\omega_{e}$, and $\tau_{W}(t)$ is a function of $\zeta(t)$. Hence, the equation of the motion would in the ideal case of a monochromatic wave be a function of $V, \omega, \psi_{e}, H_{z}$ and $T_{z}$.

\section{D. actuators forces}

The ship also reacts to the actions of its actuators, fins and rudders here. In this case the forces have the following expression :

$$
\tau_{A}=-M_{F A} \ddot{\eta}-D_{F A} \dot{\eta}+B_{F} \beta
$$

where $\beta$ is the actual position of the actuators; the matrices $M_{F A}, D_{F A}$ and $B_{F}$ are functions of the geometry of the fins and rudder and of their position on the hull. $D_{F A}$ is proportional to the speed of the $\operatorname{ship}(V)$ whereas $B_{F}$ is proportional to the square of the speed $\left(V^{2}\right)$ [6], [5], [7].

\section{E. Ideal, synthesis and simulation models}

1) Ideal model: The final ideal model should be:

$$
\bar{M}\left(\omega, \psi_{e}, V\right) \ddot{\eta}+\bar{D}\left(\omega, \psi_{e}, V\right) \dot{\eta}+G \eta=B\left(V^{2}\right) \beta+\tau_{W}\left(\omega, H_{z}, T_{z}\right)
$$

where $\bar{M}\left(\omega, \psi_{e}, V\right)=M+M_{A}\left(\omega, \psi_{e}, V\right)+\quad M_{F A}$, $\bar{D}\left(\omega, \psi_{e}, V\right)=D_{A}\left(\omega, \psi_{e}, V\right)+D_{F A}(V)$ and $B\left(V^{2}\right)=B_{F}\left(V^{2}\right)$.

\footnotetext{
${ }^{1}$ Data is obtained numerically by dedicated softwares based on strip theory or singularity sources distribution.
}

Note that what has been obtained here is a "theoretical" model depending on $V, \omega, \psi_{e}, H_{z}$ and $T_{z}$, and also naturally on the ship load. It remains theoretical because the swell is never monochromatical but may be seen as the superposition of an infinite number of elementary waves moving in different directions.

Note that if it is possible to approximate such swells in the simulations knowing the spectrum (2), it will not possible to take into account all its characteristics in a model dedicated to control law synthesis since it is considered that no sensors giving $\omega, H_{z}$ and $T_{z}$ are available in the applied study (section IV).

2) Synthesis Model: The synthesis model that will be used in section IV has been obtained by specific techniques for a frigate type vessel. Its expression could be understood from the previous detailed expression under the following assumption: the encounter angle of the swell is $90^{\circ}$, the load is known and invariant, the ship essentially reacts at its own resonant frequency, function of the load. Moreover the dynamics of the actuators is modelled by a 2 nd order LTI system.

For the control law synthesis, a linear state space model will be used with state $x=[v, p, r, \phi, \psi, \beta, \dot{\beta}]^{T}$ where $v, p$, $r$ are respectively the sway, roll and yaw velocity and $\beta$ is the actual position of the actuators. The control, $u$, is the desired position of the actuators. The measures $y$ considered for control are the roll velocity $p$ and the heading angle $\psi$. The model is of the following type:

$$
\begin{aligned}
\dot{x} & =A(V) x+B u \\
y & =C x
\end{aligned}
$$

Only the coefficients of the matrice $A$ are dependent on $V$ as second (fins and rudders efficiency), first (damping) or zeroth (buoyancy) order polynomials. The synthesis model for the studied case of section IV is finally only parameterised in speed $V$.

3) Simulation Model: To obtain the simulation model an output disturbance $w(t)$ has been added to $y$; it is nothing but the effect of the input perturbation $\tau_{W}(t)$ due to the swell, on the output. It is computed from the spectrum (2) with $T_{z}=7.1 \mathrm{~s}$ and $H_{s}=3.25 \mathrm{~m}$ (Sea State 5 in the northern Atlantic).

$$
y=\left(p+p_{w}, \psi+\psi_{w}\right)^{\top}
$$

In addition, the simulations takes into account the temporal non-linear aspects of saturation (in angle and rate for both the fins and rudders) and digitalisation of the control law. Moreover, a pure delay is added in temporal simulations to make up for the information transportation effects in the ship internal network.

\section{THE PROPOSED CONTROL LAW SYNTHESIS METHODOLOGY}

\section{A. Introduction}

Section II-E.1 showed the ideal dependence of the motion of the ship on its speed $(V)$ and on the environment 
characterized by the sea state parameters $\omega, \psi_{e}, H_{z}$ and $T_{z}$. It clearly appears that gain scheduled controllers are an interesting way to tackle the reduction of the roll induced by the swell. In general, controllers implemented in reality are PID-type. They are generally tuned at the ship roll frequency [8], [1], and finally designed with the use of optimisation. The dependance on ship speed is rarely described [1], but it may be assumed that the dependance is inversely proportionnal to the square of the speed. No theoretical proof of the closed loop stability exists in this case; but simulation tests "show in practice" the stability of the closed loop and the efficiency of the method.

In this study it is proposed to use the recent $H_{\infty} / \mathrm{LMI}$ techniques to compute gain scheduled controllers for Linear systems with varying parameters since they guarantee the closed loop stability. This section will propose a methodology for the general considered problem. In the next section, its application to particular conditions will be detailed and the performances of the obtained regulator will be compared to those of PIDs.

\section{B. The general specifications for the control law synthesis}

Specifications characterizing the desired behavior of the ship are, from the point of view of mechanics and comfort:

- reduce the roll motion inside the swell bandwidth and do not amplify it outside ! On a military vessel, reduction will be crucial during some technical operations but of less importance during others. So compromise, to save power for example, lead to introduce a tuning parameter to define a roll reduction quality,

- keep yaw angle as constant as possible (yaw and roll motions are coupled),

- do not use to much energy,

- respect a given power repartition on the actuators (fins and rudders). They have different effects on the ship motions. The fins are used only for roll stabilisation, and should interfere very little with the heading. On the contrary, rudders have a great influence on roll motions, but are primary used to control the yaw,

- tolerate only "acceptable" position and speed saturation of the actuators.

Others specifications are added in a control engineering point of view:

- the closed loop and the controller must be stable.

- some robustness properties are necessary against uncertainties like delay, discretisation...

\section{A four step methodology}

In order to derive gain scheduled controllers from the now classical $H_{\infty} /$ LMI techniques, one needs a linear parameterically varying standard model defined from the dynamic model of the ship (section II) and weight functions, which must be a translation of the previous specifications. Note that the main difficulty here is to translate the realistic specifications in a mathematical manner: weights of a $H_{\infty}$ standard model. It is proposed to decompose it into two: first, compute the weights for fixed values of the varying parameters; then, compute the varying standard model with an interpolation technique.

More generally, the following four-stage methodology is proposed to achieve the final synthesis goal:

-Stage 1: Determine the varying parameters, which will be considered [gridding]. All the varying parameters of the idealized model introduced in section II-E.1 should be considered; but the more parameters, the more complicated will be the computation; moreover, it is sometimes impossible to take into account all these parameters in a synthesis model (see section II-E.2 for the studied case model).

-Stage 2: Choose the parameters values as a grid of well chosen values. At each set of the parameters, determine the weights for the standard model that result in a $H_{\infty}$ controller such that specifications are fulfilled. This is based on the resolution of a multi-objective optimisation problem.

-Stage 3: Compute, with a classical interpolation technique, a linear standard model with varying parameters from the fixed standard models, results of stage 2. The choice of stage 2 may be revised following the achieved interpolation errors: less "optimal solutions" may be chose so as to allow good interpolation to take place.

-Stage 4: Compute a gain scheduled controller for the linear varying parameters model with standard code (Matlab's LMI toolbox, for example).

Some of these technical points are now classical: the resolution of an $H_{\infty}$ control problem (stage 2), and of the $H_{\infty}$ /LMI gain scheduled controller (stage 4) is possible with standard Matlab code. The definition and resolution of a multi-objective optimisation problem (stage 2) to obtain optimised weights were already introduced in [9] in the roll stabilisation context and also present (under a different form) in [10]. But in [9] only controllers for fixed configurations were obtained. The specificity of this paper lays in the whole methodology, which considers completely the variation of the parameters.

In the next section, an application of this general methodology is developed.

\section{APPLICATION OF THE METHODOLOGY IN THE STUDIED CASE}

The studied ship is a frigate-type ship (length $120 \mathrm{~m}$, displacement 3000 metric tons). The considered environmental conditions are sea state $5\left(T_{z}=7.1 \mathrm{~s}\right.$ and $\left.H_{s}=3.25 \mathrm{~m}\right)$ for a encounter angle $\psi_{e}$ of $90^{\circ}$. The models for synthesis and simulation are described in section II-E.2 and II-E.3.

\section{A. The varying parameters}

Two parameters were used: the ship speed over the water, and a tuning parameter called Stabilisation Quality Factor (SQF). Dependance in the ship speed is obviously needed 
due to the importance of the efficiency changes of the actuators (see section II-E.2). The SQF defines roll reduction quality, and corresponds to the depth of the roll sensibility transfer function. Its value is intended to be directly tuned from the bridge or by an adaption process - taking into account energy consumption, actuators' saturation levels, sea state measurements.

The values of the parameters are defined by a comprehensive gridding, with steps every 5 knots in speed from 10 to 25 knots, and every 1 unit in SQF quality from 2 to 8 .

\section{B. Controller synthesis for fixed parameters}

A more comprehensive study [9], which is the premise for the present article, gives the details for the computation of controllers for static values of the parameters. The method used was to solve a multi-objective optimisation problem with an evolution strategy (genetic algorithm), and to choose a particular solution from strict guidelines. Another solution was used here, though very close in principles to the former for which the optimisation process took quite some time to reach a good values. Now the use of comprehensive exploration of the parameters' space gives good results in relatively little time.

1) Definition of the multi-objective optimisation problem: The choice of the control law (here the weights of the $H_{\infty}$ standard problem) is defined as a multi-objective optimisation problem under contraints. The objectives and contraints are derived from the specifications introduced in subsection III-B:

O1 : Reduce the roll motion. It is expressed as the minimisation of the roll RMS value on a particular sea state for the closed loop system.

$\mathrm{O} 2$ : Use the minimal quantity of energy. It is necessary to ensure that the two actuators do not compensate for one another, case which may appear (for MIMO PID, for instance). The sum of the RMS values of the fins' and rudders' positions (resp. $\sigma_{\alpha}$ and $\sigma_{\delta}$ ) is minimised, for the same sea-state.

O3 : Respect as precisely as possible the repartition constraint. The objective is defined as the weighted ratio 'use of the fins' over 'total use of the actuators', the weights being the $H_{\infty}$ norm of the open loop transfer functions between fin and rudder position and roll.

The constraints used, in addition to these objectives, are:

C1 : the controller must be stable;

C2 : the closed loop (system + controller), given a control application delay, must be stable;

C3 : the delay margin must be acceptable, in order to take into account the digitalisation induced phase (and delay), the information transfer delay... The delay margin is evaluated with sensibility and complementary sensibility output transfer functions analysis, as the control problem is MIMO - see [11], [12];
C4 : the amplification under and over the ship roll resonance must be low [13]; They are calculated from sensitivity transfer between $p_{w}$ and $p$;

C5 : the actuators may not endure too much saturation, in both position and velocity. It is not possible to determine exactly the saturations levels when working in the frequency domain, for it has only a temporal meaning. Yet, they can be evaluated from statistical considerations ([1], [14]).

Note that this optimisation framework can be used to tune different controllers. It is proposed to tune $H_{\infty}$ controllers, but it has also been used for PID for comparison.

Solutions were chosen after a comprehensive exploration of the parameter space following a "design of experiments" process. The use of genetic algorithms (GA) may lead to better results [9], but since the time spent at tuning controllers was at stake, GA were put aside.

2) Definition of the $H_{\infty}$ problem: It is set up as a mixed sensitivity problem (see figure 1). $H_{\infty}$ synthesis induces the resolution of an optimisation problem. It results in the controller $K(s)(u=K y)$ such that the $H_{\infty}$ norm of the closed loop transfer from $w$ to $z$ is below an acceptable level.

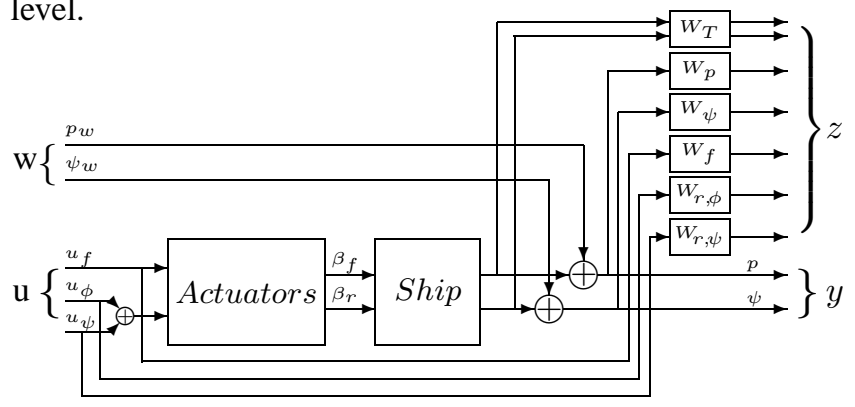

Fig. 1. Mixed sensitivity problem with output additive disturbance.

Weights transfer functions (see figure 1) are defined as follows:

- $W_{p}$ is the roll derivative sensitivity weight. Its shape is characterised by the depth (or SQF) of its well, centered about the roll resonance frequency of the ship (see figure 2). The SQF varies between 2 and 8 ;

- $W_{\psi}$ is the yaw sensitivity weight. Its expression is of the form $W_{\psi}(s)=K_{\psi} \frac{\tau_{\psi 1} s+1}{\tau_{\psi 2} s+1}$, with $\tau_{\psi 1}<\tau_{\psi 2}$;

- $W_{f}$ and $W_{r \phi}$ are the fin and rudder position sensitivity weights in their use for roll attenuation; theirs expressions are $W_{f(, r \phi)}(s)$ $=K_{f(, r \phi)} \frac{\left(\tau_{f n 1(, r \phi n 1)} s+1\right)\left(\tau_{f n 2(, r \phi n 2)} s+1\right)}{\left(\tau_{f d 1(, r \phi d 1)} s+1\right)\left(\tau_{f d 2(, r \phi d 2)} s+1\right)} \quad$ with $\tau_{f n 2(, r \phi n 2)}<\tau_{f d 2(, r \phi d 2)}<\tau_{f d 1(, r \phi d 1)}<$ $\tau_{f n 1(, r \phi n 1)}$;

- $W_{r \psi}$ is the rudder position sensitivity weight in its use for heading-control; its expression is of the form $W_{r \psi}(s)=K_{r \psi} \frac{\tau_{r \psi 1} s+1}{\tau_{r \psi 2} s+1}$ with $\tau_{r \psi 1}<\tau_{r \psi 2}$. Note that the rudder control is divided in two components with separate bandwidth defined by the characteristics of $W_{r \phi}$ and $W_{r \psi}$; 
- $W_{T}$ is the complementary sensitivity weight; This is a low pass filter with expression of the form $W_{T}(s)=K_{T}\left(\frac{\tau_{T 1} s+1}{\tau_{T 2} s+1}\right)^{3} I_{2}$, with $\tau_{T 1}<\tau_{T 2}$. This weight is tuned so that the delay margin has a given minimal value (derived from [11]); it also keeps the controller bandwidth under reasonnable values (though this is not clearly expressed in the controller specifications).

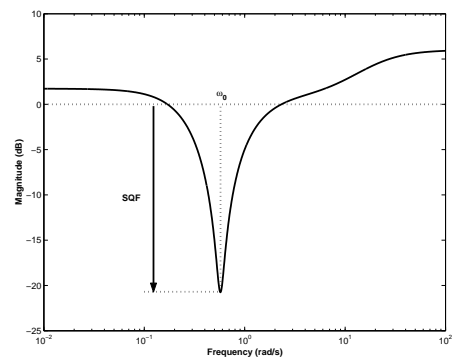

Fig. 2. The roll derivative sensitivity $W_{p}$.

Most of the parameters of these weights have a fixed value. In fact, only $K_{f}$ and $K_{r \phi}$ are variables ${ }^{2}$ in the multiobjective optimisation problem.

3) The results of the "controller synthesis for fixed parameters" step: The results of this step are not the $H_{\infty}$ controllers, even if they have to be calculated in order to assess their performances. The real results are the weights themselves and the standard models, for every combination of parameters. They are the basis for the next step.

\section{Computation of the LPV model}

The computed standard models (dynamics augmented with frequency weights) show a dependency on $V$ ( $V$ and $V^{2}$ actually) and $S Q F$. With a classical interpolation technique [15], it is possible to synthesise their expression in the following LPV model:

$$
\begin{aligned}
P\left(V, V^{2}, S Q F\right) & =\left[\begin{array}{ccc}
A\left(V, V^{2}, S Q F\right) & B_{1}\left(V, V^{2}, S Q F\right) & B_{2} \\
C_{1}\left(V, V^{2}, S Q F\right) & D_{11} & D_{12} \\
C_{2} & D_{21} & D_{22}
\end{array}\right] \\
& =P_{c}+P_{V} V+P_{V^{2}} V^{2}+P_{S Q F} S Q F
\end{aligned}
$$

A basic solution is to consider the model (13) as an affine model $P_{a}\left(X_{1}, X_{2}, X_{3}\right)$ obtained by replacing $V$ by $X_{1}, V^{2}$ by $X_{2}$ and $S Q F$ by $X_{3}$. The parameters $X_{1}, X_{2}$ and $X_{3}$ are supposed to be independent of each other ${ }^{3}$. This leads to a very conservative model.

A polytopic model has instead been used. There exists dedicated control law synthesis code 4 . The chosen model is put under the following form:

$$
P_{p}(\theta)=\left\{\sum_{i=1}^{7} \alpha_{i}(\theta) \pi_{i}, \alpha_{i}(\theta) \geq 0, \sum_{i=1}^{7} \alpha_{i}(\theta)=1\right\}
$$

\footnotetext{
${ }^{2}$ This may appear very simple, yet this is the result of several tests ! ${ }^{3}$ with $X_{1} \in[1025], X_{2} \in\left[\begin{array}{ll}100 & 625\end{array}\right]$ and $X_{3} \in[28]$.

${ }^{4}$ LMI toolbox of matlab, for example.
}

where the vertices $\pi_{i}$ are the image of the vertices of a polytopic domain $\mathcal{P}$ which define acceptable restricted values of $\theta$ : $\left\{\theta=\left(X_{1}, X_{2}, X_{3}\right) / \theta=\sum_{i=1}^{7} \alpha_{i} P_{i}, \alpha_{i} \geq 0, \sum_{i=1}^{7} \alpha_{i}=1\right\}$. The vertices $P_{i}$ of $\mathcal{P}$ have been chosen such that the possible values of $\left(V, V^{2}, S Q F\right)$ of the initial LPV model (13) are included in $\mathcal{P}$ (see figure 3 ). The facet $\left\{P_{5}, P_{6}, P_{7}\right\}$ express the constraint that for small values of $V$ no good roll damping is possible ${ }^{5}$. The influence of $V^{2}$ remains sufficently low to keep representation adequate (without too much conservatism).

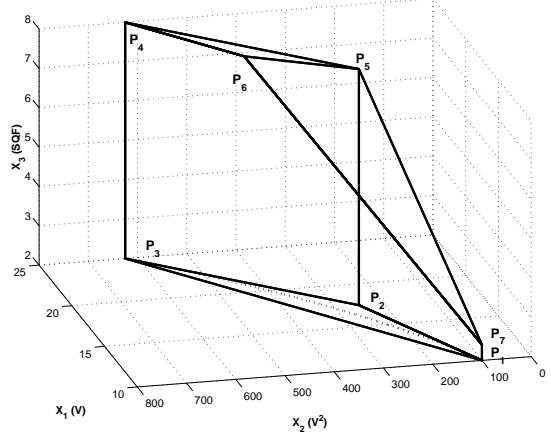

Fig. 3. Polytope $\mathcal{P}$ (solid) and projection on $\left(X_{1}, X_{2}\right)$ (dotted) of all possible $\left(V, V^{2}, S Q F\right)$ triplets.

\section{Computation of the gain scheduled controller}

Once a valid polytopic standard model is written, the computation of the polytopic controller is quite straightforward. The LMI Control toolbox of Matlab provides tools for such a work. It constitutes a attractive aspect from an industrial point of view.

A quite similar method, and giving potentially better results, is to represent the LPV system with a LFT [2], [15]. The controller synthesis is clearly described in the literature (and now quite classical), yet it requires a great quantity of computation and formalisation. Furthermore, controllers may exist without the theorem (and the equations) allowing to compute it.

\section{E. Simulation results and comparisons}

1) Discrete form controller: For the application of the controller in simulation, its matrices are computed at each time step, a discretisation done with a zero order hold approximation (it requires the computation of a matrix exponential): this method is quite non optimal, but works properly on a $800 \mathrm{MHz}$ computer, and it ensures stability of the controller.

2) Comparisons: Four controllers were tested in simulation: a static $H_{\infty}$ controller (IV-B.2), a MIMO PID obtained with similar technique as IV-B.1, the polytopic $H_{\infty}$ controller and a gain scheduled SISO PID tuned with methodology from [1]. Table I gives stabilisation

\footnotetext{
${ }^{5}$ This is the result of Stage 2 .
} 
performances (RMS roll reduction rate) for sea state 5 $\left(\psi_{e}=90^{\circ}\right)$. It shows the advantage of $H_{\infty}$ controllers over PID controllers. Unsurprisingly, the LMI method for gain scheduled $H_{\infty}$ controllers introduces conservatism and the final results do not comply with our objectives as good as the static $H_{\infty}$ controller. This stems from the synthesis technique in itself: il involves solving LMI for each summit of the polytope (see [16]) with a single $X$ matrix, whereas for a $H_{\infty}$ synthesis the same matrix solves only one LMI.

\begin{tabular}{|c|c|}
\hline PID & $75.5 \%$ \\
Static $H_{\infty}$ & $77.2 \%$ \\
\hline \hline Varying PID & $59.9 \%$ \\
Polytopic $H_{\infty}$ & $70.3 \%$ \\
\hline
\end{tabular}

TABLE I

PERFORMANCES AT 24 KNOTS - RMS ROLL REDUCTION RATE.

3) Simulations: The controller proves its interest in simulations when conditions vary and on rougher sea states than the one used in the controller synthesis.

Figure 4 presents temporal signals for a varying ship speed $\left(\psi_{e}=90^{\circ}\right.$, sea state $6-T_{z}=8.5 \mathrm{~s}$ and $\left.H_{s}=5 \mathrm{~m}\right)$ : at $\mathrm{t}=200 \mathrm{~s}$ the speed, $V$, is increased from 10 to 20 knots. Two simulations are presented: when the controller adapts to the actual ship speed, and when it remains adapted for 10 knots. The benefit of speed adaptation is clear : energy saving (look at $\alpha$ and $\beta$, the fin and rudder angles of attack).
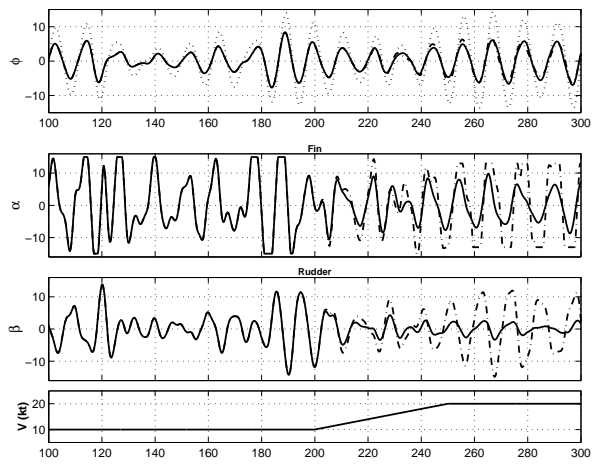

Fig. 4. Roll signal without stabilisation system: dotted; with speed adapted stabilisation (SQF 2): solid; with stabilisation adapted to 10 knots (SQF 2): dash-dotted. $\alpha$ and $\beta$ stand for fin and rudder angle of attack.

Figure 5 presents temporal signals for a varying SQF (with conditions: sea state $6, V=24$ knots and $\psi_{e}=75^{\circ}$ ). It shows the interest of the possibility to make SQF vary to avoid saturation.

\section{CONCLUSION-PERSPECTIVES}

A methodology is given for computing a gain scheduled controller, based on LMI and on polytopic modelisation of the process. Parameters used are the ship speed and a stabilisation quality factor. The methodology has been applied to a frigate-like ship in simulation. Evolution of the parameters during the simulation is possible, which
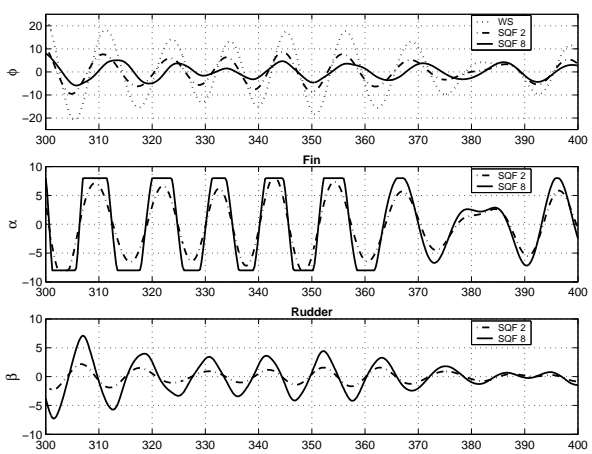

Fig. 5. Roll signal without stabilisation system (WS): dotted; with stabilisation (SQF 2): dash-dotted; with stabilisation (SQF 8): solid; $\alpha$ and $\beta$ stand for fin and rudder angle of attack.

influence the controllers matrices. Better performance than with usual PID are obtained. However results suffer from conservatism introduced by the polytopic synthesis. Further research involves using LFT modelisation and introducing more parameters.

\section{REFERENCES}

[1] A. Lloyd, Seakeeping, Ship Behaviour in Rough Weather, ser. Marine Technology. Hellis Horwood, 1989.

[2] P. Apkarian and P. Gahinet, "A convex characterization of gainscheduled $h_{\infty}$ controllers," IEEE TAC, vol. 40, no. 5, pp. 853-864, 1995.

[3] H. Tanguy and G. Lebret, "Stabilisation en roulis d'un navire de type frégate," JESA, vol. 37, no. 2, pp. 287-302, 2003.

[4] T. I. Fossen, Navigation and Guidance of Ocean Vehicles. New York: John Wiley \& sons, 1994

[5] J. Sgobbo and M. Pearsons, "Rudder/fin roll stabilization of the uscg wmec 901 class vessel," Marine Technology, vol. 36, no. 3, pp. 157170, july 1999.

[6] J. Dern, G. Hardier, and J. Jung, "Modélisation hydrodynamique et lois de commande du système de stabilisation du porte-avions à propulsion nucléaire," in Actes de la conférence ATMA, 1987.

[7] H. Tanguy and G. Lebret, "Stabilisation en roulis d'un navire de type frégate," in Actes de la conférence CIFA 2002, Nantes, France, july 2002.

[8] M. Katebi, N. Hickey, and M. Grimble, "Evaluation of fin roll stabiliser controller design," in 5th IFAC Conference on Manoeuvering and Control of Marine Crafts, Aalborg, Danmark, 23-25 august 2000.

[9] H. Tanguy, G. Lebret, and O. Doucy, "Multi-objective optimisation of pid and $h_{\infty}$ fin/rudder roll controller," in Proceedings of MCMC 2003, Girona, Spain, september 2003.

[10] G. Duc and A. Hiret, "Application au pilotage d'une chaîne de tangage d'un missile sur un large domaine de vol," APII-JESA, no. 35 , pp. 107-125, 2001

[11] B. Bergeon, F. Cazaurang, and N. Philippe, "H-infinity design and multivariable delay margin," in SSC 2001, Prague, Tcheck Republic, 2001.

[12] S. Cluzel, "La marge de retard multivariable, dea," Université de Nantes, 2000

[13] G. Hearns, R. Katebi, and M. Grimble, "Robust fin roll stabiliser controller design," in 5th IFAC Conference on Manoeuvering and Control of Marine Crafts, Aalborg, Danmark, 23-25 august 2000.

[14] W. Price and R. Bishop, Probabilistic Theory of Ship Dynamics. Londres: Chapman and Hall, 1974.

[15] J. Magni, Linear Fractional Representations with a Toolbox for Use with MATLAB, Toulouse, France, 12 2001, technical report TR 240/2001 DCSD.

[16] J.-M. Biannic, "Commande robuste des systèmes à paramètres variables. applications en aéronautique," Ph.D. dissertation, SUPAERO, Toulouse, France, 1996. 